Article

\title{
Cu(II)/Guanidine Functionalized Disiloxane Complex of Supramolecular Structures for Visible Light-Driven Photocatalysis of Congo Red
}

\author{
Maria E. Fortună ${ }^{1}$, Lucia Pricop ${ }^{1}$, Mirela Zaltariov ${ }^{1}$ (D), Dumitru Popovici ${ }^{1}$, Maria Ignat ${ }^{1,2, *(\mathbb{D})}$, \\ Valeria Harabagiu ${ }^{1, *(D)}$ and Bogdan C. Simionescu ${ }^{1}$ \\ 1 "Petru Poni" Institute of Macromolecular Chemistry, 41A Grigore Ghica Voda Alley, 700487 Iasi, Romania; \\ fortuna.maria@icmpp.ro (M.E.F.); pricop.lucia@icmpp.ro (L.P.); zaltariov.mirela@icmpp.ro (M.Z.); \\ dumitru.popovici@icmpp.ro (D.P.); bcsimion@icmpp.ro (B.C.S.) \\ 2 Faculty of Chemistry, “Alexandru Ioan Cuza” University of Iaşi, 11 Bd. Carol I, 700506 Iaşi, Romania \\ * Correspondence: ignat.maria@icmpp.ro (M.I.); hvaleria@icmpp.ro (V.H.)
}

check for

updates

Citation: Fortună, M.E.; Pricop, L.; Zaltariov, M.; Popovici, D.; Ignat, M.; Harabagiu, V.; Simionescu, B.C. $\mathrm{Cu}$ (II)/Guanidine Functionalized Disiloxane Complex of Supramolecular Structures for Visible Light-Driven Photocatalysis of Congo Red. Polymers 2022, 14, 817. https://doi.org/ $10.3390 /$ polym 14040817

Academic Editor: Sudip Chakraborty

Received: 31 January 2022

Accepted: 18 February 2022

Published: 20 February 2022

Publisher's Note: MDPI stays neutral with regard to jurisdictional claims in published maps and institutional affiliations.

Copyright: (C) 2022 by the authors. Licensee MDPI, Basel, Switzerland. This article is an open access article distributed under the terms and conditions of the Creative Commons Attribution (CC BY) license (https:// creativecommons.org/licenses/by/ $4.0 /)$.

\begin{abstract}
The present study focuses on the synthesis of a new guanidine-functionalized disiloxane used as a ligand to obtain copper(II) complexes linked through hydrogen bonding into supramolecular structures. A two-step procedure was used to prepare the guanidine functionalized disiloxane ligand. Firstly, the hydrosilylation reaction between the siloxane precursor, namely 1,1,3,3tetramethyldisiloxane (DS), and the allyl glycidyl ether (AGE) was performed in the presence of a platinum catalyst resulting in glycidoxypropyldisiloxane (DS-PMO) intermediary compound. In the second step, DS-PMO derivative was modified with 1,1,3,3-tetramethyl guanidine (TMGu) to obtain the guanidine-functionalized disiloxane ligand (bGu-DS) that was further used for the coordination of copper(II) acetate hydrate. The structures of the ligand and of its $\mathrm{Cu}$ (II) complex were confirmed by spectral methods (IR, UV-Vis, NMR, XRF) and correlated with theoretical calculations using semiempirical PM6 and DFT methods. The copper(II) complex was found to exhibit low optical band gap energy $(2.9 \mathrm{eV})$ and good photocatalytic activity under visible light irradiation in the decomposition of Congo Red (CR). A dye removal efficiency higher than $97 \%$ at the catalyst and CR concentrations of 1 and, respectively, $200 \mathrm{mg} / \mathrm{L}$ was obtained.
\end{abstract}

Keywords: copper(II) complex; guanidine functionalized disiloxane ligand; Congo red; visible light driven photocatalysis

\section{Introduction}

Increasing stressing environmental concerns at the international level are related to water pollution generated by different sources of human activity. One of the most polluted wastewaters is recorded for the textile industry due to refractory dye compounds, mainly resulting from the dyeing and finishing processes. The dyes are hardly biodegradable or even non-degradable and very toxic for environmental systems. Over time, dye removal from textile effluents has been achieved by the use of chemical, physical and biological methods [1,2] and sustained research efforts are dedicated to the topic. The photodegradation of dyes in the presence of a UV and/or visible photoactive catalyst is one of the currently investigated techniques. Nowadays, researchers worldwide are fully focused on the use of heterogeneous photocatalysis for environmental remediation and wastewater treatment [3-5]. Usually, in heterogeneous photocatalytic processes, photon energy is used by a solid catalyst and converted into chemical energy, being really effective in the degradation of a wide range of organic contaminants. Over time, semiconductors $\left(\mathrm{TiO}_{2}, \mathrm{Cu}_{2} \mathrm{O}, \mathrm{ZnO}, \mathrm{BiVO}_{4}, \mathrm{CdS}, \mathrm{WO}_{3}\right.$ and so on) were intensively studied and applied in the photochemical degradation of various organic pollutants [6]. Recently, copper-based photosensitizers have been proved to be efficient in light-induced 
processes due to their great reducing power and long lifetime in an excited state [7]. Furthermore, considerable attention has been given to the visible-light photoactive copper-based photocatalysts [8,9].

Possible ligands for copper cations are guanidine derivatives. Due to their unusual donor properties, guanidines are able to coordinate transition metal ions with different oxidation states giving discrete metal complexes or extended structures. In particular, bis(guanidines) form binuclear compounds self-assembled in supramolecular coordination polymers by using different reaction conditions and/or intermolecular interactions: hydrogen bonding, $\pi-\pi$ stacking, van der Waals forces, etc. Since 2000, a variety of copper complexes have been reported by using bis(guanindines) with ethylene and propylene backbone sequences which showed catalytic activity in atom transfer radical polymerization and oxidation catalysis. [10,11].

On the other hand, polysiloxanes are attractive catalyst supports as they are highly hydrophobic and possess excellent surface properties [12]. As a consequence of their unusual flexibility ( $T_{g}=-123{ }^{\circ} \mathrm{C}$ for polydimethylsiloxane), these polymers are able to expose the side chain catalytic centers to the reagents through an easy modification of their conformation. Due to their inorganic skeleton, polysiloxanes are also chemically and thermally stable (except in the presence of strong acids and bases). In addition, their organic side substituents can be easily modified [13] offering flexibility in the choice of ligand groups $[14,15]$. Usually, the synthesis of various classes (linear, branched, brushes, dendrimers) of polysiloxanes allows the tailoring of the structure according to the requirements of each specific process. Thus, polysiloxanes were studied either as matrices for polymer/photocatalyst composites [16] or as supports for transition metal catalysts [12-17]. For example, $\mathrm{TiO}_{2}$ photoactive compound was embedded into polydimethylsiloxane matrices and the resulting hydrophobic material was studied as photocatalyst in the decomposition of different dyes [18].

Due to the role transition metals played in the performance of molecular materials, remarkable catalytic properties with specific applications in photocatalysis could be obtained [19]. Among other materials, such as $\mathrm{ZnO}$ [20] or tridoped $\mathrm{TiO}_{2}$ [21], copper oxides and complexes are also known to show electronic transitions in the visible region [22,23]. Many polymers supported copper complexes for the treatment of environmental contaminants were proposed [24]. Moreover, ligand-functionalized polysiloxanes complexed with transition metals were also described [12-17], but none of them were applied in photocatalytic processes.

Considering the advantages of polymer-supported catalysts, such as their higher durability and selectivity and their easy separation from the reaction products, in this study a new guanidine modified disiloxane was synthesized. Its amphiphilic copper complex was proved to possess a polymeric supramolecular structure based on hydrogen bonding. It was expected to expose the guanidine- $\mathrm{Cu}$ polar moieties toward the interface between the solid complex and dye aqueous solution, and to exhibit great activity in visible light-driven photocatalytic processes. The molecular structure, morphology and physicochemical properties of the synthesized complex have been investigated by using infrared (FTIR) UV-Vis and ultraviolet-diffuse reflection (UV-DR) spectroscopies, scanning electron microscopy (SEM), differential scanning calorimetry (DSC), and thermo-gravimetric analysis (TGA), respectively. The FTIR experimental data were compared with theoretical calculations. Moreover, the synthesized copper(II) complex has been involved as a photocatalyst in the photodegradation experiment of Congo Red $(\mathrm{CR})$, knowing the limited use of this anionic diazo dye in textile industry due to its high toxicity [25].

\section{Materials and Methods}

\subsection{Materials}

1,1,3,3-Tetramethyldisiloxane 97\% (DS), allyl glycidyl ether 99\% (AGE), Karstedt catalyst, 1,1,3,3-tetramethylguanidine 99\% (TMGu), copper(II) acetate monohydrate, 
n-hexane, and Congo Red dye $\left(\mathrm{C}_{32} \mathrm{H}_{22} \mathrm{~N}_{6} \mathrm{Na}_{2} \mathrm{O}_{6} \mathrm{~S}_{2}\right)(\mathrm{CR})$ were purchased from Aldrich and used as received. Toluene (Aldrich) was dried over sodium wire and distilled before use.

\subsection{Preparation of bis(Guanidine Functionalized)-1,1,3,3-Tetramethyldisiloxane Ligand (bGu-DS)}

The bGu-DS ligand was obtained in a two-step procedure previously described for the synthesis of a guanidine monofunctionalized glycidoxypropyltrisiloxane [26] (see details in Sections S1 and S2 (Supplementary Materials)). Shortly the hydrosylilation reaction of DS with AGE afforded bis(3-propoxymethyloxirane)-1,1,3,3-tetramethyldisiloxane (DS-PMO) that was further reacted with TMGu to yield the bGu-DS ligand, as a viscous oily liquid.

\subsection{Complexation of Copper with $b G u-D S$}

To obtain the copper complex (Cu-bGu-DS), bGu-DS (3.314 mmol) in $\mathrm{C}_{2} \mathrm{H}_{5} \mathrm{OH}(20 \mathrm{~mL})$ was stirred at room temperature until complete dissolution and then $\mathrm{Cu}\left(\mathrm{CH}_{3} \mathrm{COO}\right)_{2} \times \mathrm{H}_{2} \mathrm{O}$ (7.57 mmol) (0.93 mmol excess) was added. After $2 \mathrm{~h}$ of stirring at room temperature, a fine precipitate appeared and the reaction mixture turned turquoise. The precipitate was separated from the reaction mixture by centrifugation at $3000 \mathrm{rpm}$. To the resulting deposit, $10 \mathrm{~mL}$ of $1 / 1(v / v)$ ethanol/distilled water mixture were added in order to extract under stirring traces of non-reacted copper acetate. After a second centrifugation, the solid was introduced in a vacuum oven at room temperature to remove the solvent traces. A turquoise waxy material was obtained in $85 \%$ yield.

\subsection{Photocatalytic Activity}

We used $50 \mathrm{mg}$ of copper(II) complex of guanidine functionalized disiloxane (CubGu-DS) catalyst for all experiments. The catalyst was in contact either with $50 \mathrm{~mL}$ of dye solutions (solid/liquid ratio (s/1), 1/1 w/v) of different CR concentrations (50, 100 and $200 \mathrm{mg} / \mathrm{L}$ ) or with $100 \mathrm{~mL}$ CR solution (s/1 ratio, $0.5 \mathrm{w} / \mathrm{v}$ ) of $100 \mathrm{mg} / \mathrm{L}$ CR concentration. The prepared mixtures containing solid catalyst and dissolved dye were stirred first in the dark for $60 \mathrm{~min}$ in order to achieve the adsorption equilibrium. Afterward, a lowpressure sodium lamp $(400 \mathrm{~W}$, producing a virtually monochromatic light averaging a $589.3 \mathrm{~nm}$ wavelength and a power density of $14.5 \mathrm{~mW} / \mathrm{cm}^{2}$, at a distance between lamp and solution surface of $15 \mathrm{~cm}$ ), was turned on, and the photocatalytic experiment started. The experiment lasted for $100 \mathrm{~min}$, and from time to time aliquots were collected, filtered and the concentration of CR dye was checked at the specific wavelength of $498 \mathrm{~nm}$ by using a Shimadzu $2400 \mathrm{UV}$-Vis spectrophotometer, versus a prior registered calibration curve in the range of $0-20 \mathrm{mg} / \mathrm{L} \mathrm{CR}$ concentrations. In order to verify whether the CR dye molecule was not affected/decomposed by the visible light irradiation used, a blind photolysis experiment was performed in the absence of the photocatalyst.

\subsection{Measurements}

Infrared spectra (FTIR) were obtained by using a Nicolet 60 SX FT-IR (Thermo Fisher Scientific, Waltham, MA, USA) under dry air, at room temperature, on KBr pellets, in the range of $4000-400 \mathrm{~cm}^{-1}$ or in the far IR region.

${ }^{1} \mathrm{H}-\mathrm{NMR}$ and ${ }^{13} \mathrm{C}-\mathrm{NMR}$ spectra were recorded on a Bruker Avance III 400 spectrometer (Bruker Company, Billerica, Massachusetts) in $\mathrm{CDCl}_{3}$.

The presence and ratio of metal and $\mathrm{Si}$ were evidenced using an energy-dispersive X-ray fluorescence (EDXRF) system EX-2600 X-Calibur SDD (Xenemetrix Ltd., Migdal Haemek, Israel).

Thermogravimetric data (TGA) were registered on STA 449F1 Jupiter equipment (Netzsch Company, Selb, Deutschland), in the temperature range of $30-700{ }^{\circ} \mathrm{C}$, under airflow $(50 \mathrm{~mL} / \mathrm{min})$ with a heating rate of $10{ }^{\circ} \mathrm{C} \mathrm{min}^{-1}$. 
Differential scanning calorimetry (DSC) measurements were conducted on a DSC 200 F3 Maia device (Netzsch Company, Selb, Deutschland) at heating and cooling rates of $10{ }^{\circ} \mathrm{C}$ $\mathrm{min}^{-1}$, under nitrogen atmosphere, at a flow rate of $50 \mathrm{~mL} \mathrm{~min}^{-1}$.

A Quanta 200 scanning electron microscope (FEI Company Hillsboro, OR, USA) was used to identify the surface morphology. The measurements were performed in a dry nitrogen atmosphere avoiding water absorption.

UV-Vis measurements were performed on a Shimadzu UV-2400 spectrophotometer (Shimadzu Company, Kyoto, Japan) in DMF solution. For solid state analysis an ISR-2200 integrating sphere attached to the spectrophotometer was used, allowing the registration of UV-DR spectra on $\mathrm{MgO}$ pellets as white standard.

\section{Results and Discussion}

A new guanidine difunctionalized disiloxane ligand, bGu-DS, was synthesized in a high yield through a two-step procedure, previously described for the preparation of a guanidine monofunctional trisiloxane [26]. The details of the synthesis and structure assessment of bGu-DS and of the intermediate product, namely bis(3-propoxymethyloxirane)1,1,3,3-tetramethyldisiloxane (DS-PMO) are presented in Sections S1 and S2 (Supplementary Materials).

The as-obtained bGu-DS amphiphilic ligand was further used for the complexation of $\mathrm{Cu}$ (II) cations (Scheme 1), with the expectation to obtain a photocatalyst active in the visible light region. The composition and the molecular structure of $\mathrm{Cu}-\mathrm{bGu}-\mathrm{DS}$ complex were investigated by XRF, FTIR and UV-Vis spectroscopic techniques. The results obtained confirmed the theoretical calculations, while the photocatalytic efficiency of the $\mathrm{Cu}(\mathrm{II})$ complex under visible light irradiation was checked on aqueous solutions of Congo Red dye.<smiles>CO[Si](C)(C)CCCOCC(O)CN=C(C)N(C)C</smiles>

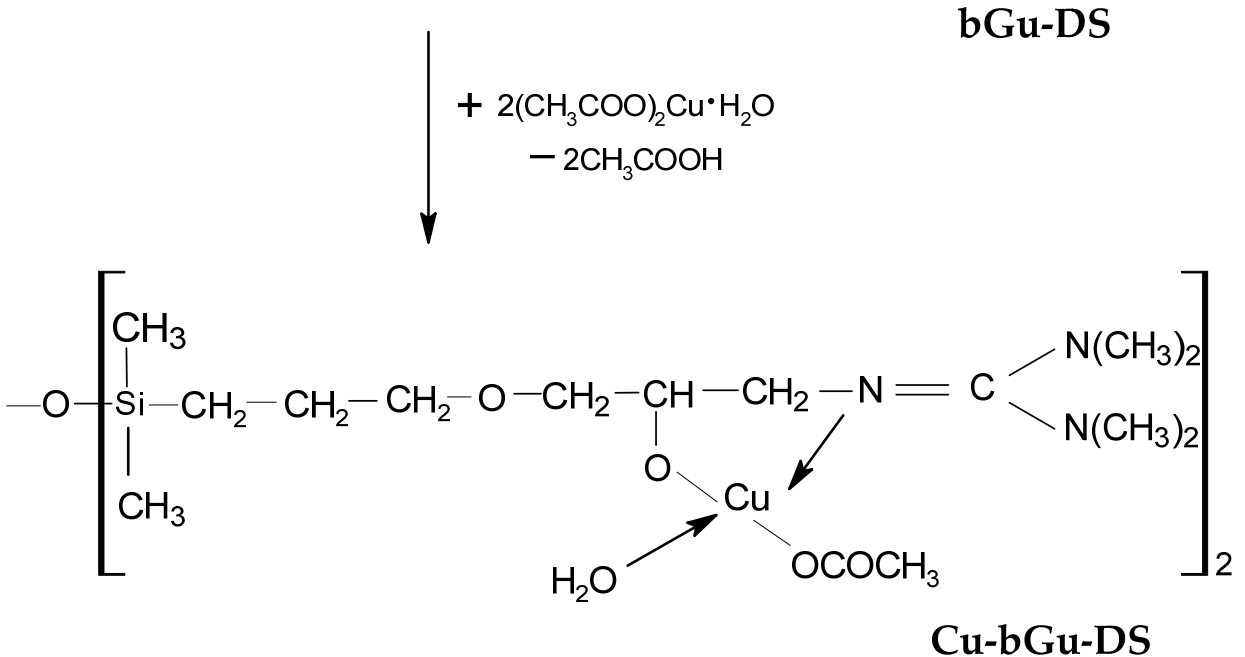

Scheme 1. Synthesis of $\mathrm{Cu}-\mathrm{bGu}-\mathrm{DS}$.

\subsection{Structural Characterization of $\mathrm{Cu}-b \mathrm{G} u$-DS Complex}

3.1.1. X-ray Fluorescence (XRF) and Fourier Transform Infrared (FTIR) Investigation

The composition and the structure of $\mathrm{Cu}-\mathrm{bGu}-\mathrm{DS}$ complex depicted in Scheme 1 were first assessed by using XRF and FTIR spectroscopies. The XRF spectrum of Cu-bGu-DS compound (Figure 1) shows the presence of both $\mathrm{Si}$ and $\mathrm{Cu}$ species. A Si/Cu atomic ratio of 
$1 / 1.03$, close to the $1 / 1$ theoretical value was calculated from XRF data with the following relation:

$$
\frac{S i}{C u}=\frac{\frac{\text { Counts }_{S} * N_{A}}{A_{S i}}}{\frac{\text { Counts }_{C u} * N_{A}}{A_{C u}}}
$$

where, Counts $S_{S i}$ and Counts $_{C u}$ are the line intensities of $S i$ and $C u$ atoms, respectively; $N_{A}$ is the Avogadro's number; $A_{S i}$ and $A_{C u}$ are the atomic masses of $S i$ (28.085 a.u.) and $C u$ (63.546 a.u.), respectively.

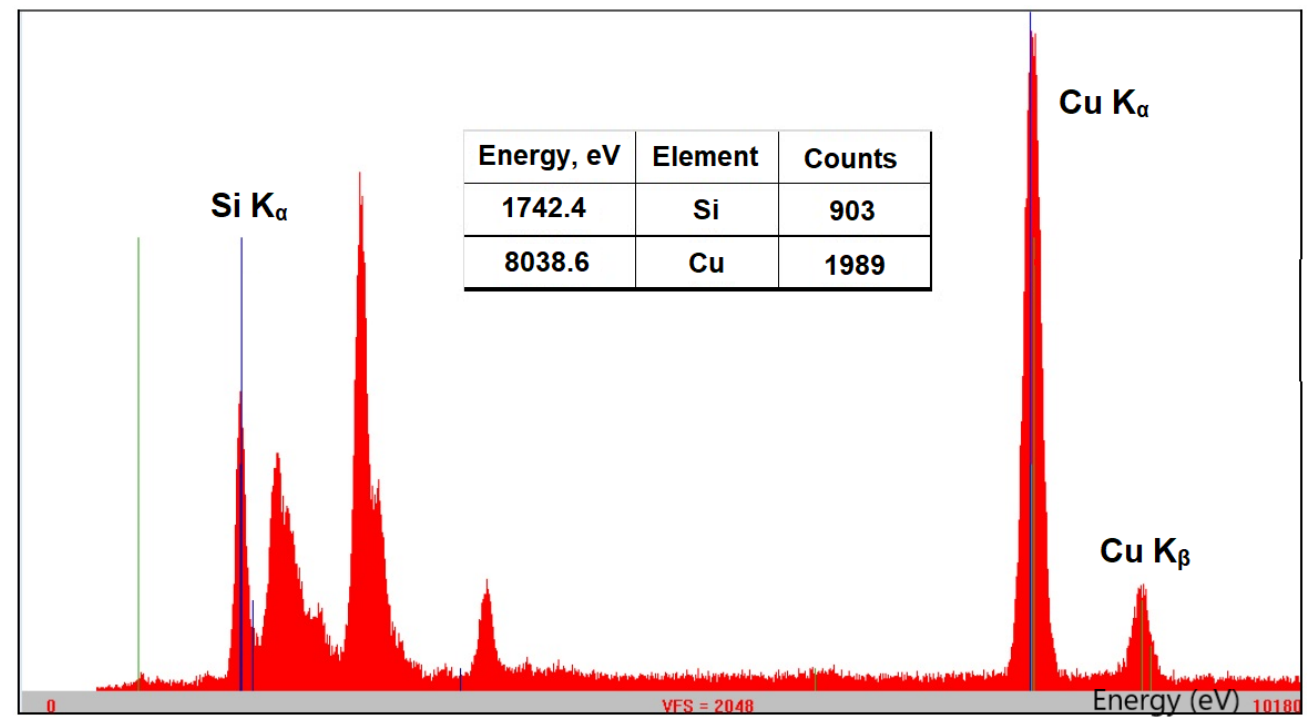

Figure 1. X-ray fluorescence (XRF) spectrum of $\mathrm{Cu}-\mathrm{bGu}$-DS complex showing the $\mathrm{K} \alpha$ lines associated with the transition of an electron from the $\mathrm{L}$ to $\mathrm{K}$ shell for $\mathrm{Si}$ and $\mathrm{Cu}$ atoms and the $\mathrm{Kb}$ of $\mathrm{Cu}$ (due to the transition of an electron from $\mathrm{M}$ to $\mathrm{K}$ shell); inset table: energy (eV) and intensity (counts) of Si and $\mathrm{Cu}$ specific transitions.

FTIR absorptions of bGu-DS ligand and of its copper(II) complex are depicted in Figure 2. As one may see from Figure 2a, both compounds present characteristic bands for C-H asymmetric and symmetric stretching vibrations between $2860-2960 \mathrm{~cm}^{-1}$, and different vibrations of methyl, methylene and $\mathrm{C}-\mathrm{N}$ groups in the $1430-1475 \mathrm{~cm}^{-1}$ spectral interval. At $1256,841,797 \mathrm{~cm}^{-1}$ in both spectra $\mathrm{Si}_{-} \mathrm{CH}_{3}$ characteristic absorptions are visible, while the band at $1188 \mathrm{~cm}^{-1}$ and the large bands centered at 1052 (Figure 2a, top) or 1114-1045 $\mathrm{cm}^{-1}$ (Figure 2a, bottom) attributed to $\mathrm{C}-\mathrm{O}$ moieties and to the superposed band of $\mathrm{C}-\mathrm{O}-\mathrm{C}$ and $\mathrm{Si}-\mathrm{O}-\mathrm{Si}$ groups, respectively, are the fingerprint of the hydroxyether-siloxane sequence.

The FTIR spectrum of the ligand (Figure 2a, bottom) reveals a large band with two maxima centered at 3219 and $3412 \mathrm{~cm}^{-1}$ specific to secondary hydroxyl groups linked through hydrogen bonding, while the spectrum of $\mathrm{Cu}-\mathrm{bGu}-\mathrm{DS}$ complex (Figure 2a, top) also contains the band of the crystallization water $\left(3342 \mathrm{~cm}^{-1}\right)$ and short bands assigned to hydrogen bonding between the crystallization water molecules and guanidine groups (2488 and $1942 \mathrm{~cm}^{-1}$ ) that are giving rise to a supramolecular coordination polymer [27]. The band at $1755 \mathrm{~cm}^{-1}$ in the spectrum of $\mathrm{Cu}-\mathrm{bGu}$-Ds complex, attributed to the $\mathrm{C}=\mathrm{N}$ $\rightarrow \mathrm{Cu}$ group, red shifted as compared to $\mathrm{C}=\mathrm{N}$ band found at $1667 \mathrm{~cm}^{-1}$ in the bGu-DS spectrum (Figure 2a, bottom), suggests the complexation of the copper(II) cations by nitrogen atoms. Another specific band of the copper complex, attributed to the symmetric stretching vibration of the acetate group of the complex is located at $1411 \mathrm{~cm}^{-1}$. To find out the bands superposed in the region $1850-1450 \mathrm{~cm}^{-1}$, the deconvolution of the IR spectrum of Cu-bGu-DS was performed (Figure 2b). The bands at $1693 \mathrm{~cm}^{-1}$ and at $1639 \mathrm{~cm}^{-1}$ are assigned to the anion acetate group, and to the $\mathrm{OH}$ deformation vibrations, respectively, while the band at $1595 \mathrm{~cm}^{-1}$ is due to asymmetric stretching of the acetate group. The 
magnitude of separation of the acetate group $\Delta=v_{\mathrm{as}}\left(\mathrm{COO}^{-}\right)-v_{\mathrm{s}}\left(\mathrm{COO}^{-}\right)$of the bands located at $1411 \mathrm{~cm}^{-1}$ (Figure 2a, top) and $1595 \mathrm{~cm}^{-1}$ (Figure $2 \mathrm{~b}$ ) is of $184 \mathrm{~cm}^{-1}$, higher than the $\Delta$ ionic $\left(160-170 \mathrm{~cm}^{-1}\right)$, proving a monodentate coordination mode of the acetate groups by $\mathrm{Cu}(\mathrm{II})$ ions $[28,29]$, as depicted in Scheme 1.

Moreover, in the far-IR spectrum of the $\mathrm{Cu}$ (II) complex (Figure 2c) the presence of the absorption bands at 287, 253, 231 and $210 \mathrm{~cm}^{-1}$ assigned to the coordination bonds between $\mathrm{Cu}$ (II) and nitrogen/oxygen atoms also confirms the formation of $\mathrm{Cu}$-bGu-DS complex [30].
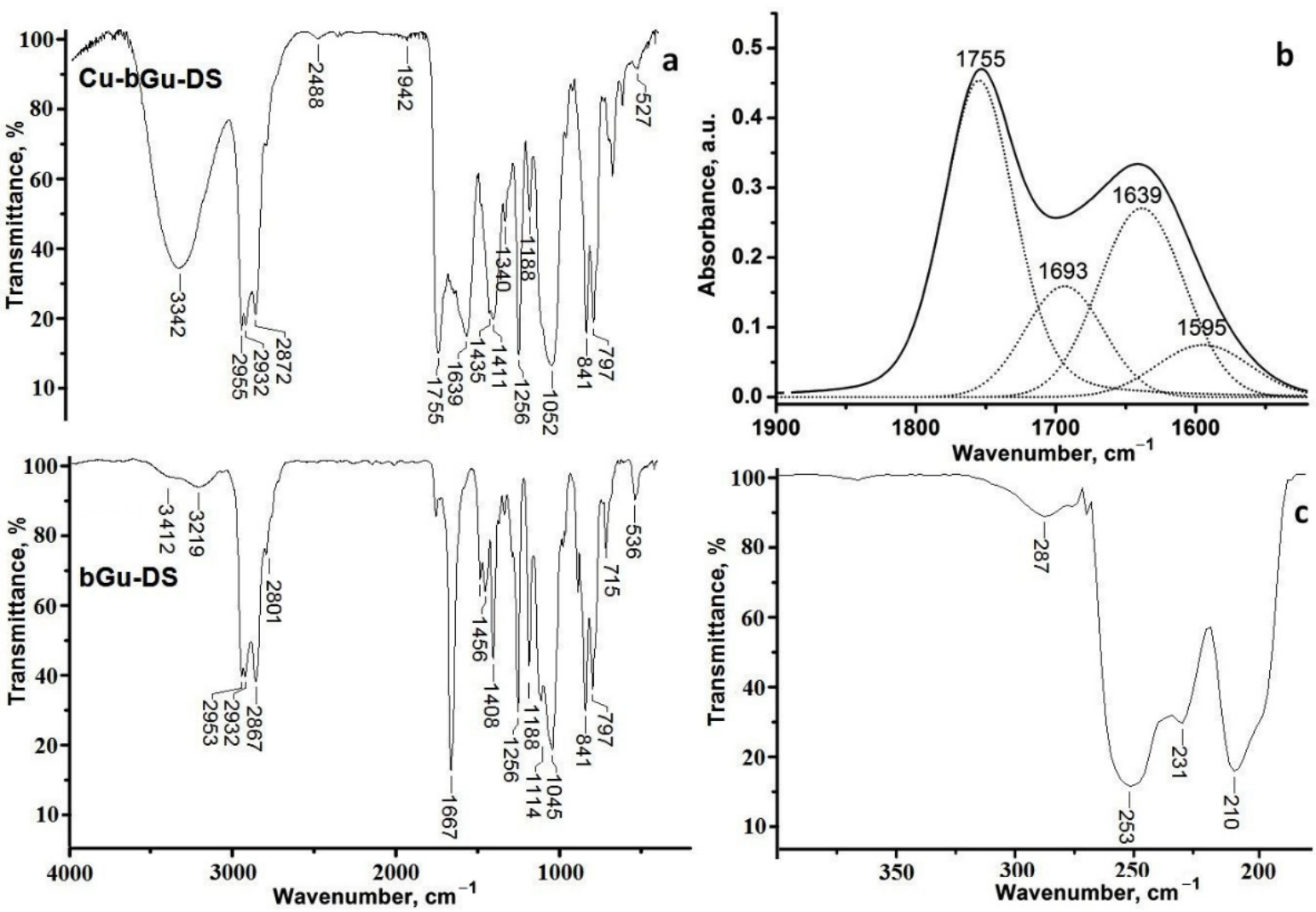

Figure 2. (a) Fourier transform infrared (FTIR) spectra of bGu-DS (bottom) and Cu-bGu-DS (top); (b) Deconvoluted IR spectrum of the Cu(II) complex; (c) far-IR spectrum of Cu-bGu-DS sample.

\subsubsection{Theoretical Study of the Conformation of Cu-bGu-DS Complex}

As the bGu-DS molecule has a symmetrical structure with two ligand functionalities linked to the disiloxane group (Scheme 1), to keep the accuracy of the calculations as well as all atoms in the ligand functional group, a simplified ligand structure (L) was used for theoretical calculations. Thus, only one of the guanidine-hydroxypropoxy-ethyl ligand functionality was kept, the other one being replaced by a methyl group (Figure 3a). The spatial conformation obtained at minimum energy for $\mathrm{L}$ and for $\mathrm{L}-\mathrm{Cu}^{2+}$ complex are shown in Figure 3b,c where the atoms are labeled as follows: white, $\mathrm{H}$; dark gray, $\mathrm{C}$; red, $\mathrm{O}$; blue, $\mathrm{N}$; orange, $\mathrm{Si}$; green, $\mathrm{Cu}$.

After the first step of minimization using PM6 as a level of theory implemented in MOPAC2016 [31], the second step of DFT calculation at B3LYP/6-31G(d,p) as a level of theory implemented in GAMESS-US(2019 R1) under Windows was performed for each model compound [32-34]. No negative frequency was found at the calculation of Hessian, which indicates that the ligand and the Cu-complex are at minimum energy. The simulated vibrational spectra in the infra-red and the far infra-red regions are presented in Figure 4. For vibrational frequency lower than $2000 \mathrm{~cm}^{-1}$ a correction factor of 0.9627 was applied, according to the literature [35]. Close values of calculated characteristic frequency for the Cu-complex (Figure 4c) and the experimental ones (Figure 2b, bottom) 
were evidenced and are also compared in Table 1 for the far-IR region. Moreover, the similitude of the experimental (Figure 2a) and simulated FTIR spectra (Figure 4) is evident, proving along with XRF and FTIR findings the proposed structure of the Cu-bGu-DS monodentate complex (Scheme 1).<smiles>CN(C)C(=NCC(O)COCCC[Si](C)(C)O[Si](C)(C)C)N(C)C</smiles>
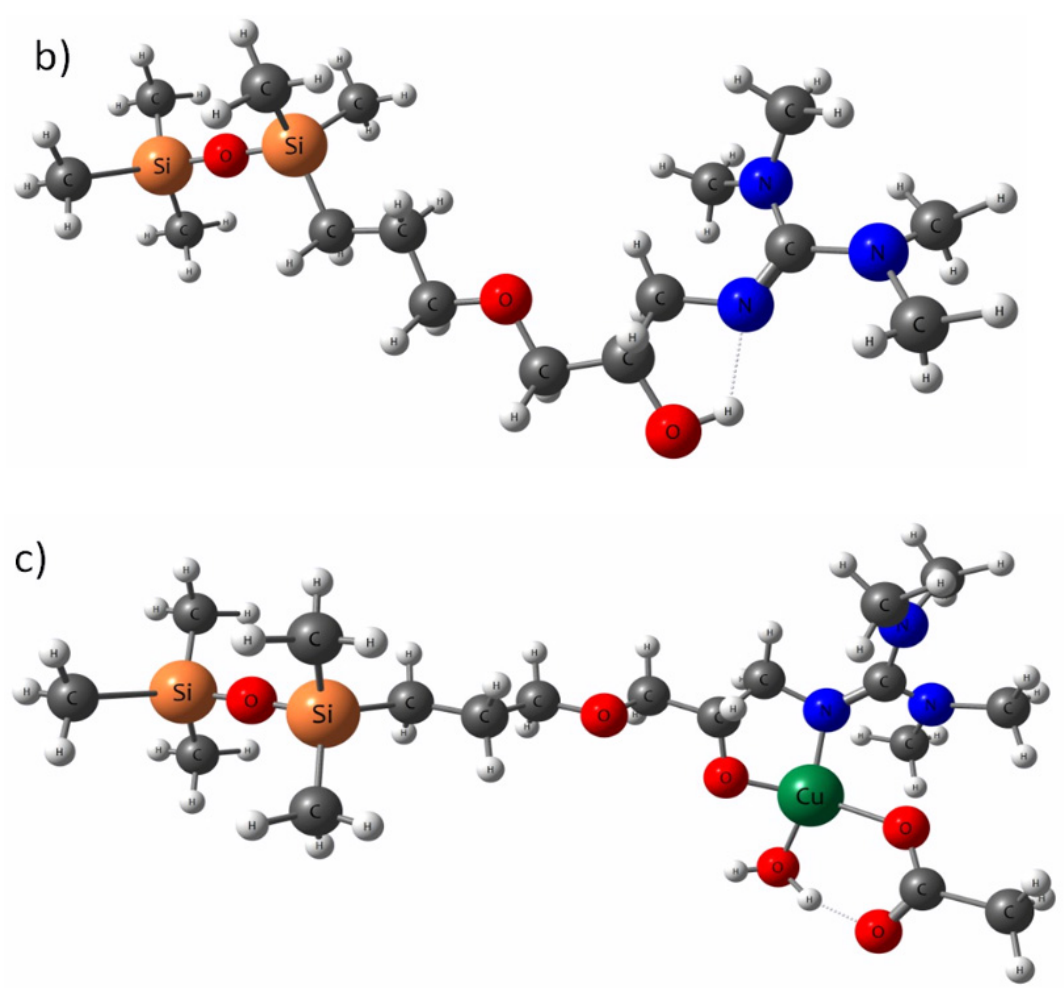

Figure 3. Simplified model structure (L) of bGu-DS ligand, (a); spatial conformation at minimum energy of $\mathrm{L}$ ligand, (b) and of $\mathrm{Cu}-\mathrm{L}$ complex, (c).

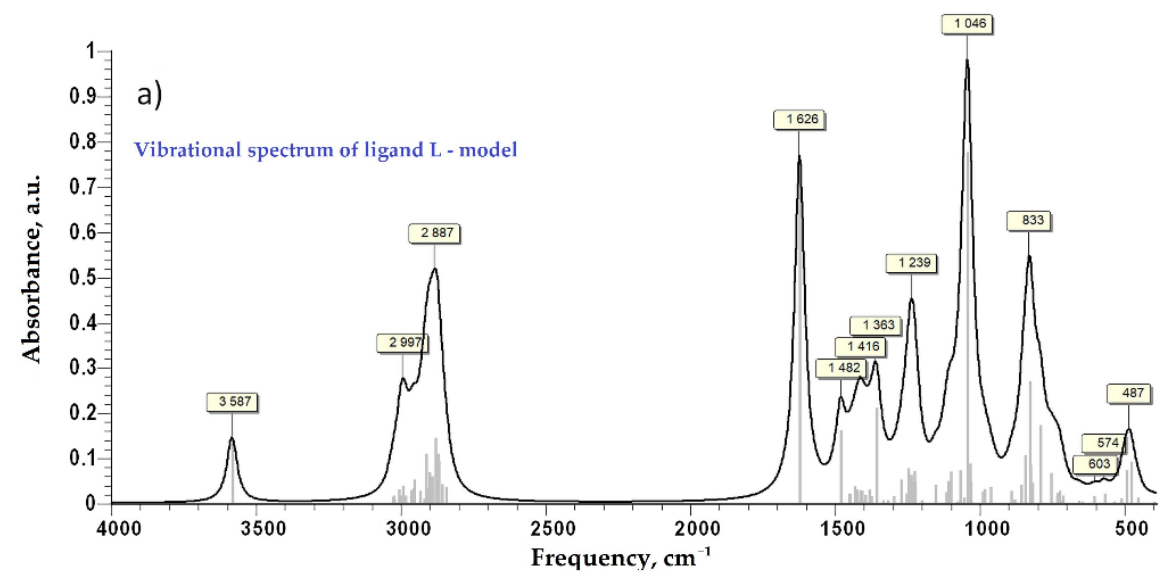

Figure 4. Cont. 

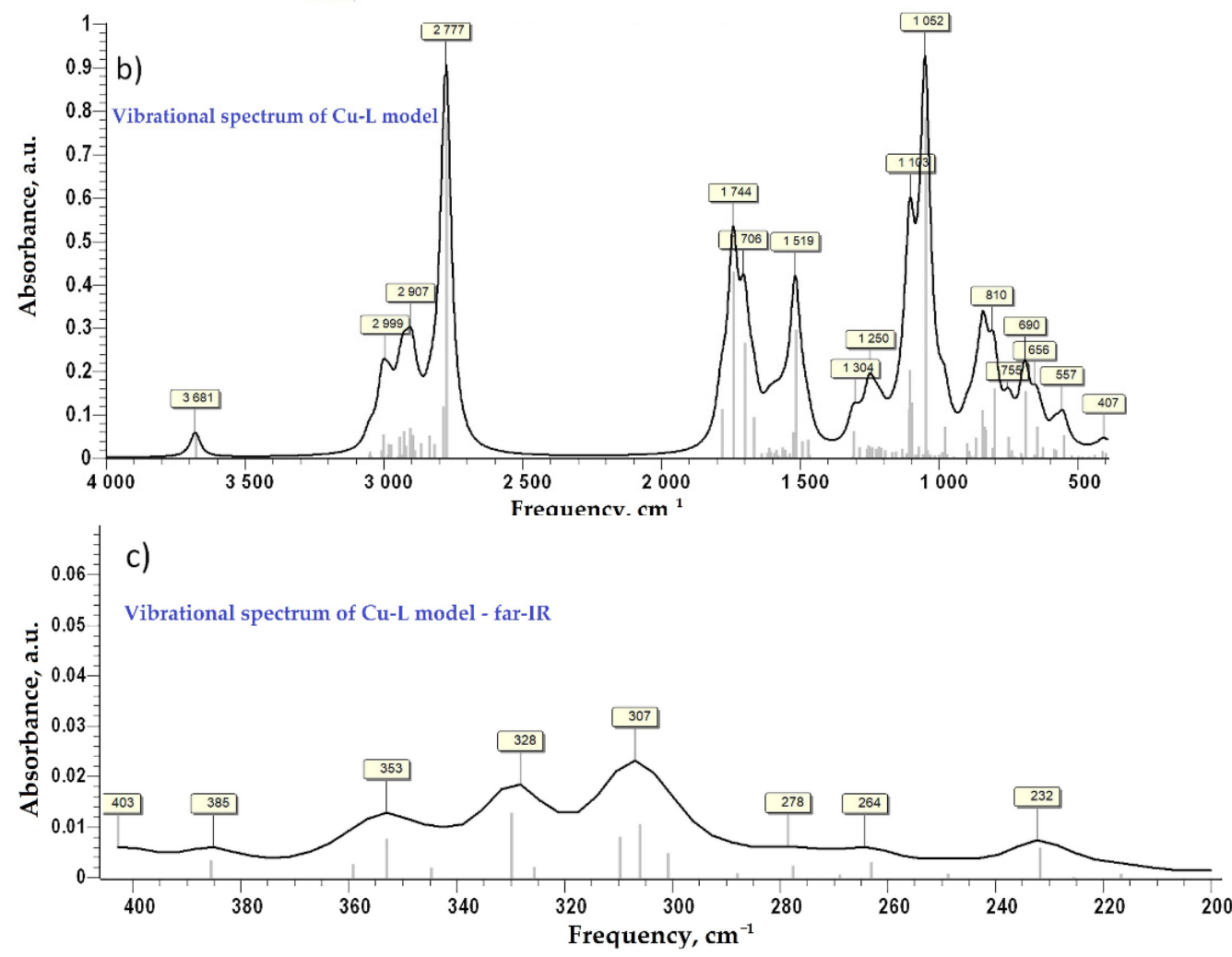

Figure 4. Vibrational spectra of the compound models in infra-red domain: vibrational spectrum of ligand model-L, (a); vibrational spectrum of Cu-L complex, (b); vibrational spectrum in far-infrared domain for $\mathrm{Cu}-\mathrm{L}$ complex, (c).

The graphical representation of fundamental HOMO and LUMO orbitals, the energies and the band gap, for both the ligand and Cu-complex are presented in Figure 5. The presence of the metallic ion strongly disturbs the localizations and energies of these orbitals. Thus, if inside the ligand they are located on the guanidine moieties, after complexation these orbitals are shifted to the atoms that participate in the formation of the complex bonds. Moreover, the energy of these orbitals strongly increases (from $\mathrm{E}_{\mathrm{HOMO}}{ }^{\mathrm{L}}=-9.009 \mathrm{eV}$ to $\mathrm{E}_{\mathrm{HOMO}}{ }^{\mathrm{Cu}-\mathrm{L}}=-5.363 \mathrm{eV}$ ), while the band gap between the fundamental state of bonding and anti-bonding orbitals decreases almost 2.5 times, from $\Delta \mathrm{E}^{\mathrm{L}}=13.676 \mathrm{eV}$ to $\Delta \mathrm{E}^{\mathrm{Cu}-\mathrm{L}}$ $=5.559 \mathrm{eV}$, remaining high enough to prevent electronic transitions that could generate emissions in the UV-Vis domain.

Table 1. Experimental and calculated absorption characteristic frequencies for the $\mathrm{Cu}$-complex in the far-IR region.

\begin{tabular}{ccc}
\hline $\begin{array}{c}\text { Experimental } \\
\left(\mathbf{( m}^{-\mathbf{1}}\right)\end{array}$ & $\begin{array}{c}\text { Calculated } \\
\mathbf{( c m}^{-\mathbf{1}} \mathbf{)}\end{array}$ & Assignment \\
\hline 210 & 216 & $\mathrm{O} \rightarrow \mathrm{Cu}-\mathrm{O}$ sym scissoring \\
231 & 232 & $\mathrm{O}-\mathrm{Cu}-\mathrm{O}$ sym scissoring \\
252 & 263 & $\mathrm{~N} \rightarrow \mathrm{Cu} \leftarrow \mathrm{O}$ asym scissoring \\
287 & 288 & $\mathrm{O} \rightarrow \mathrm{Cu}-\mathrm{O}$ sym scissoring \\
\hline
\end{tabular}



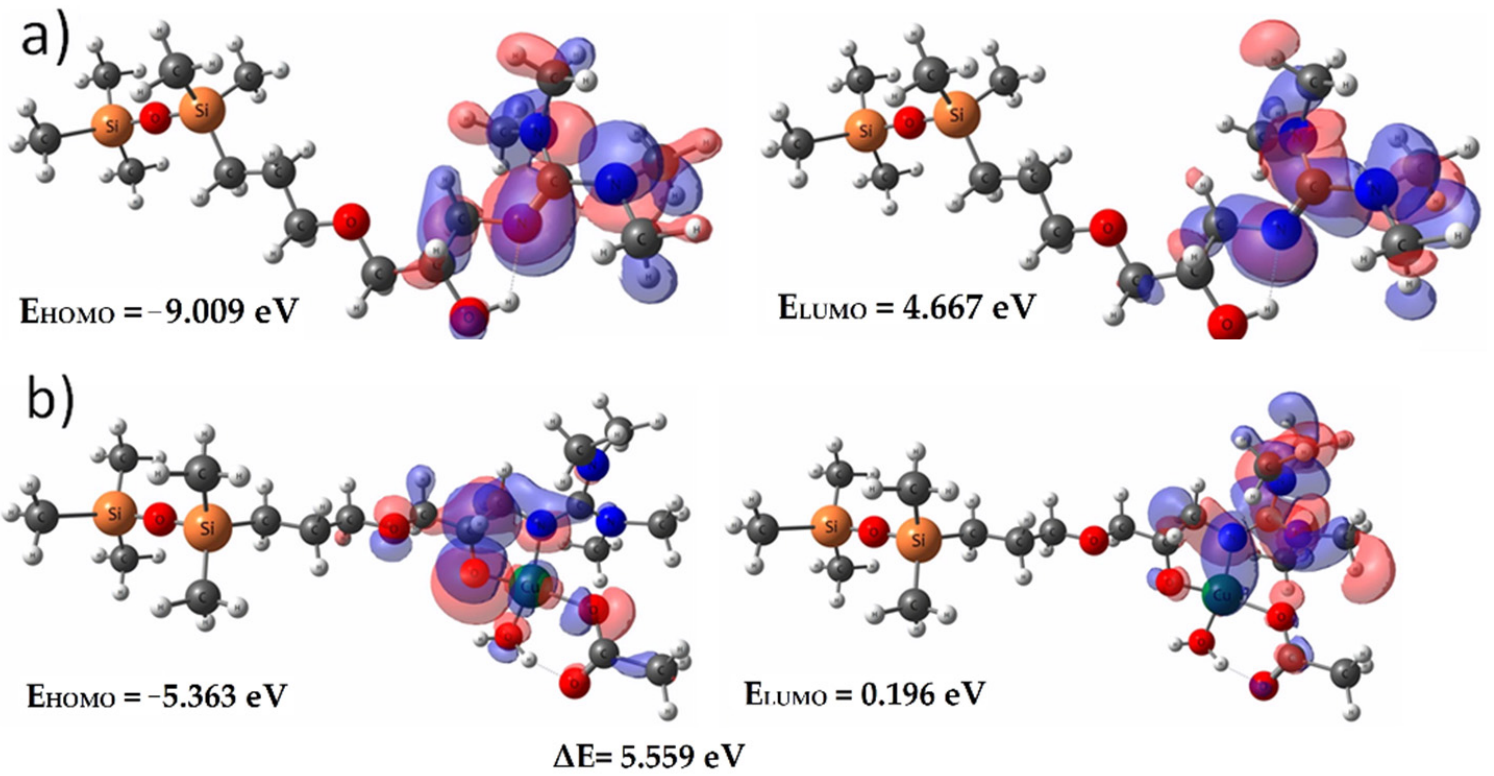

Figure 5. Fundamental HOMO and LUMO orbitals for (a) ligand model-L; (b) Cu-L complex.

\subsection{Thermal Behavior of the $b G u-D S$ Ligand and of Its $C u$ Complex}

Differential scanning calorimetry (DSC) curves (Figure 6) were registered in the temperature range between -150 and $150^{\circ} \mathrm{C}$, where only one thermal phenomenon attributed to the glass transition temperature $\left(\mathrm{T}_{\mathrm{g}}\right)$ was observed for each of the analyzed samples. The glass transition is a characteristic behavior of amorphous polymers, confirming the polymeric nature achieved through supramolecular physical hydrogen bonding, as evidenced by the FTIR spectrum of Cu-bGu-DS. As expected, a $T_{g}$ value of $-21{ }^{\circ} \mathrm{C}$ was found for $\mathrm{Cu}-\mathrm{bGu}-\mathrm{DS}$ complex, higher as compared to that identified for the bGu-DS precursor (about $-41^{\circ} \mathrm{C}$ ) due to reducing molecule flexibility, following complexation. Glass transitions at low temperatures were reported also for other compounds obtained by metal coordination with ligands having highly flexible tetramethyldisiloxane sequences in their structures [36,37].

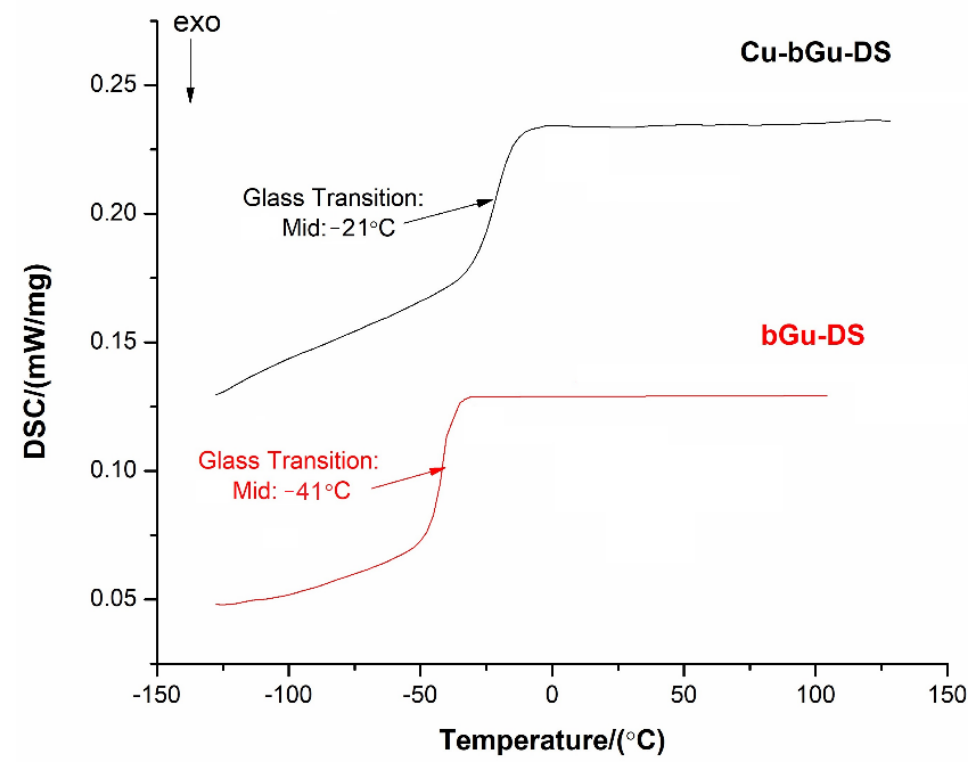

Figure 6. Differential scanning calorimetry (DSC) curves for the synthesized bGu-DS ligand and the Cu-bGu-DS complex. 
Furthermore, in order to examine the thermal stability of the prepared ligand and complex, the thermogravimetric analysis was performed in the temperature range of 20-700 ${ }^{\circ} \mathrm{C}$, under a nitrogen flow. The registered TG and DTG curves are presented in Figure 7. Three steps of weight loss, with maximum peaks at $43,182,397^{\circ} \mathrm{C}$ and at 41,180 and $377^{\circ} \mathrm{C}$ were observed in TG/DTG curves of bGu-DS ligand (Figure 7a) and Cu-bGu-DS complex (Figure $7 \mathrm{~b}$ ), respectively. At low temperatures, residual solvent and adsorbed water are supposed to leave the sample, while the next two thermal phenomena are related to the loss of structural water and to the decomposition of the ligand. At temperatures ranging between 450 and $700{ }^{\circ} \mathrm{C}$, no other decomposition phenomena were observed for both compounds up to a temperature of $700{ }^{\circ} \mathrm{C}$. The recalculated residual masses after abstraction of the losses at the first decomposition phenomena are $10.95 \%$ for the ligand and $25.44 \%$ for its complex, close to the theoretical values of $\mathrm{Si}$ content in the ligand (11.65\%), respectively to the sum of $\mathrm{Si}$ and $\mathrm{Cu}$ content in the complex (24.02). These results confirm once more the proposed structure for the $\mathrm{Cu}-\mathrm{bGu}-\mathrm{DS}$ complex.

a) TG $/ \%$

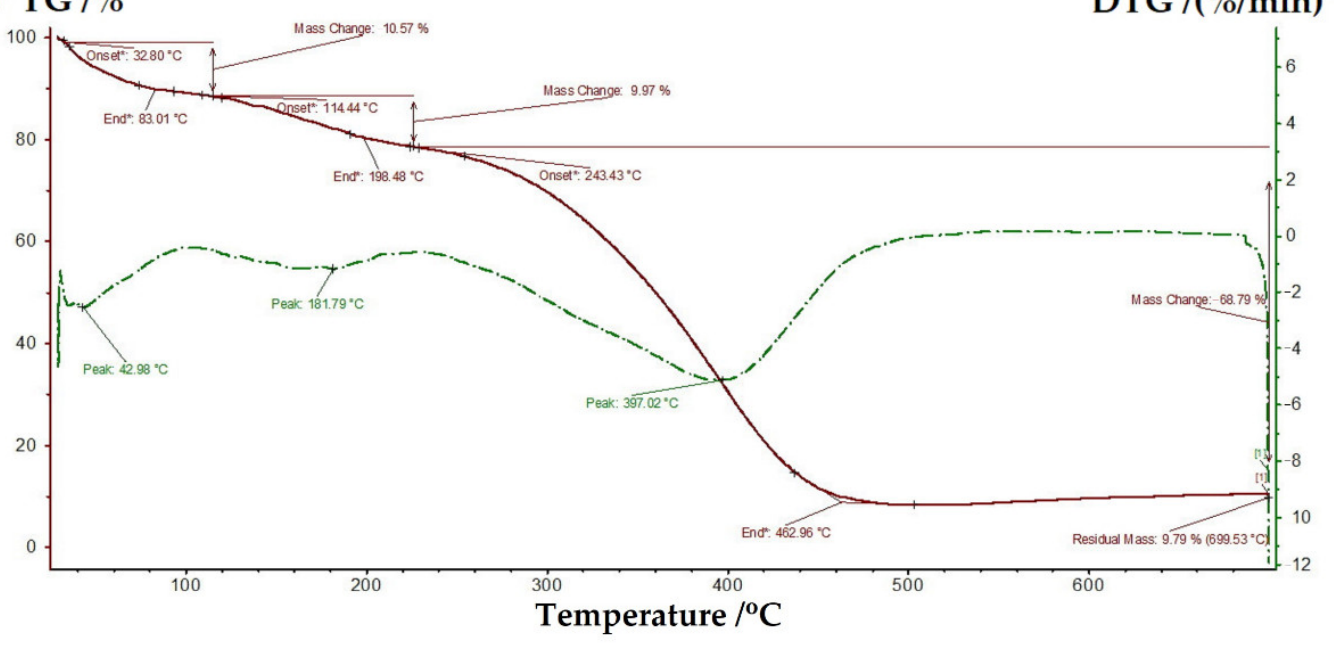

b) $\mathrm{TG} / \%$

DTG /(\%/min)

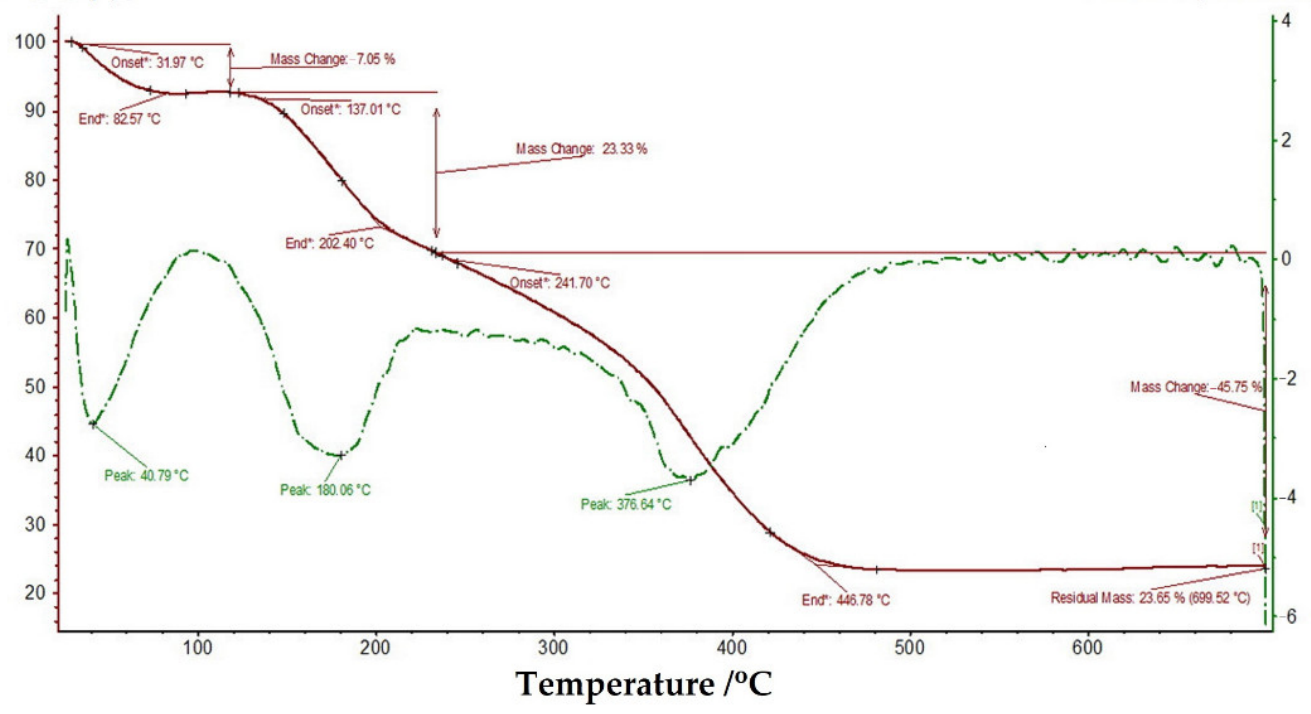

Figure 7. Thermogravimetric (TG) and differential thermogravimetric (DTG) curves of bGu-DS ligand (a) and Cu-bGu-DS complex (b). 


\subsection{Morphology Investigation of $\mathrm{Cu}-\mathrm{bGu}$-DS Complex}

The prepared $\mathrm{Cu}-\mathrm{bGu}-\mathrm{DS}$ complex was investigated from morphological point of view, and SEM and TEM images are shown in Figure 8. As can be observed, the copper complex exhibits a non-uniform inner porosity, due to which a rough surface is evidenced in the SEM image, while the TEM image shows a cauliflower-like shape of several clusters of submicronic size.
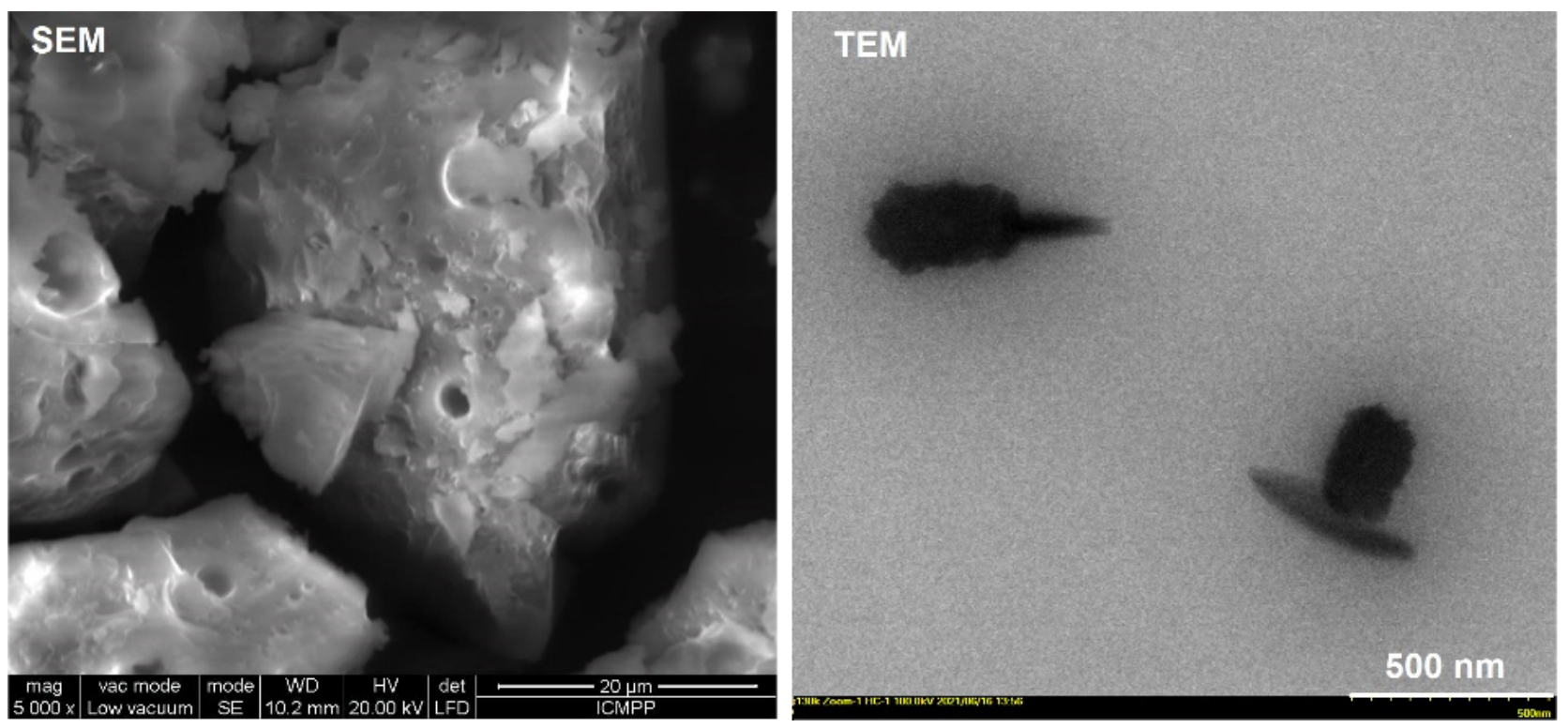

Figure 8. Scanning electron microscopy (SEM, left) and transmission electron microscopy (TEM, right) images of Cu-bGu-DS complex.

\subsection{Optical Properties of $\mathrm{Cu}-\mathrm{b} \mathrm{Gu}$-DS Complex}

UV-Vis spectra of bGu-DS and of Cu-bGu-DS compounds are shown in Figure S3 (Supplementary Materials). The ligand presents one maximum at $\lambda_{\max }=316 \mathrm{~nm}$ due to the $\mathrm{n} \rightarrow \pi$ transitions in guanidine groups, while the $\mathrm{Cu}-\mathrm{bGu}-\mathrm{DS}$ complex reveals two maxima values at $\lambda_{\max }=334 \mathrm{~nm}$ assigned to the ligand-to-metal charge transfer (LMCT) and to metal-to-ligand charge transfer (MLCT), and at $\lambda_{\max }=688 \mathrm{~nm}$ attributed to $\mathrm{d}-\mathrm{d}$ transitions.

Figure 9, displays the Tauc plots for direct and indirect allowed transitions in $\mathrm{Cu}-$ bGu-DS complex. The optical bandgap was calculated using the Tauc's theory [38] $(\alpha h \vartheta)^{n}=K\left(h \vartheta-E_{g}\right)$, where $\alpha$ is the absorption coefficient, $h$ is Planck's constant, $v$ is the frequency of the incident light, $K$ is an energy-independent parameter, $E_{g}$ is the optical bandgap, and $n=1 / 2$, and 2 for indirect and direct allowed transitions, respectively [39]. By plotting $(\alpha h \vartheta)^{1 / 2}$ against $h \vartheta$, corresponding to the direct allowed transitions of the $\mathrm{Cu}$-complex as compared to the Tauc's plot for indirect allowed transitions $\left((\alpha h \vartheta)^{2}\right.$ against $h \vartheta)$, the best linear relationship is obtained. Thus, the direct band gap has been determined by extrapolating the straight part of the plot of $(\alpha h \vartheta)^{1 / 2}$ vs. $h \vartheta$ to $h \vartheta=0$ from the intercept of the straight line at $\alpha=0$, which is found to be $2.9 \mathrm{eV}$. With such a value of the band gap energy, the prepared $\mathrm{Cu}$-bGu-DS complex seems to exhibit photocatalytic activity in the visible region. Thus, the $\mathrm{Cu}-\mathrm{bGu}-\mathrm{DS}$ complex was further subjected to photocatalytic experiments in order to evaluate its photocatalytic activity. 


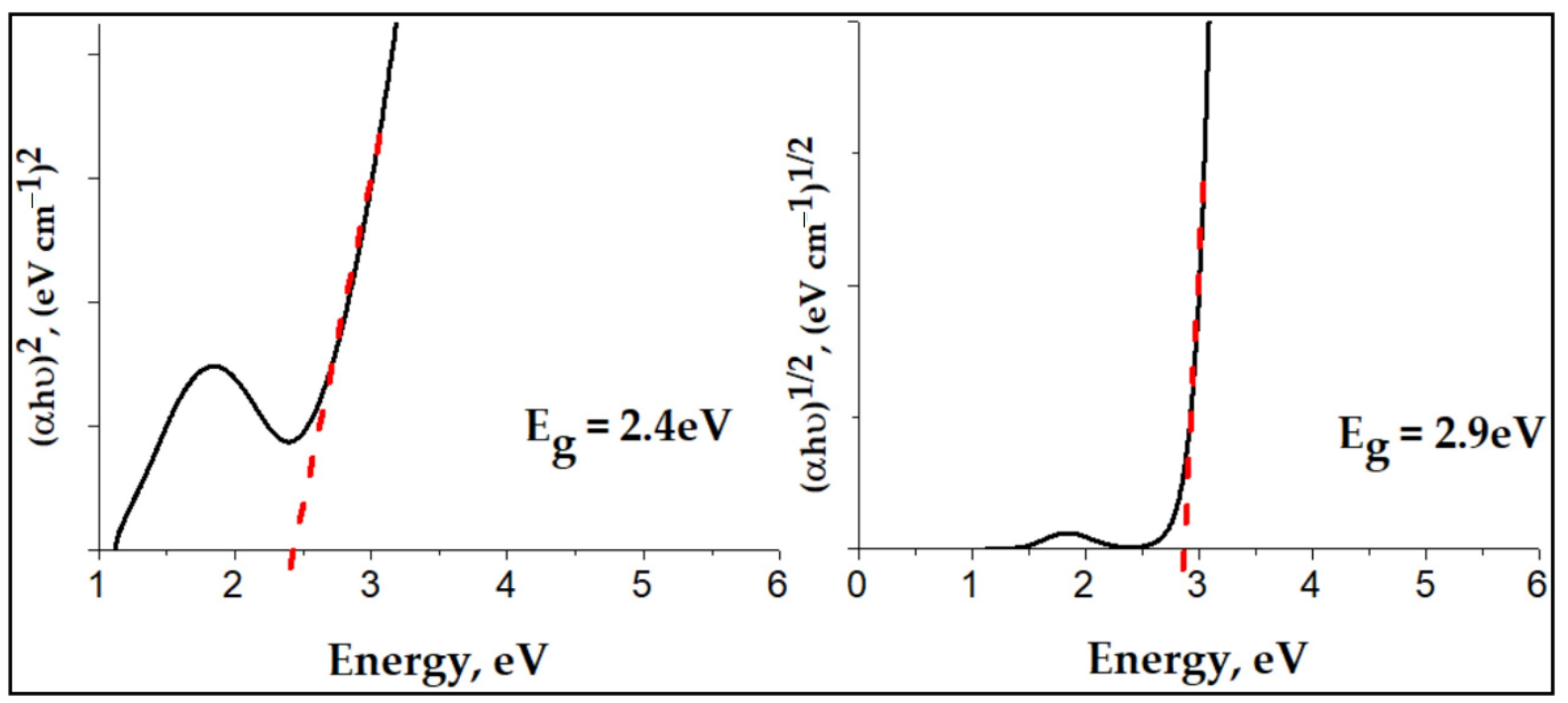

Figure 9. Tauc plot for indirect $\left((\alpha h \vartheta)^{2}\right)$ and direct $\left((\alpha h \vartheta)^{1 / 2}\right)$ allowed transitions of Cu-bGu-DS complex. Evaluation of the direct band gap energy.

\subsection{Photocatalytic Activity}

The photocatalytic experiments envisaged the use of the synthesized Cu-complex in the degradation process of Congo Red (CR) dye, under visible light $(589.3 \mathrm{~nm})$. Several experiments were performed at two solid/liquid (s/l) ratios (Cu-bGu-DS photocatalyst/CR aqueous solution of $1 \mathrm{~g} / \mathrm{L}$, and $0.5 \mathrm{~g} / \mathrm{L}$ ) and the resulting data are shown in Figure 10. Different concentrations of CR dye solution have been considered in order to find out the optimal conditions for the photocatalytic reaction. As seen in Figure 10, $\mathrm{Cu}-\mathrm{bGu}$-DS complex, due to its hydrophobic-hydrophilic structure, is able to absorb in 60 min important amounts of CR dye, varying between $25 \%$ and $100 \%$, depending on both the volume and the concentration of the dye solution. The maximum capacity in photodegradation of CR dye under visible light irradiation was found to be about $195 \mathrm{mg} \mathrm{CR} / \mathrm{g}$ of Cu-complex, at a s/1 ratio of $1 \mathrm{~g} / \mathrm{L}$ and CR concentration of $200 \mathrm{mg} / \mathrm{L}$ (Figure 10b). As can be seen from the graph, for these experimental conditions, the photocatalyst used exhibits an adsorption capacity of CR of about $25 \%$, allowing CR molecules to be concentrated around the $\mathrm{Cu}$-complex. Afterward, when the lamp was turned on, the photodegradation of $\mathrm{CR}$ molecules started with the assistance of the $\mathrm{Cu}-\mathrm{bGu}-\mathrm{DS}$ complex. During the first $5 \mathrm{~min}$ of irradiation, the degradation efficiency rose from $25 \%$ to $58 \%$, resulting in a fast degradation of adsorbed CR molecules on the surface of the synthesized photocatalyst. After this step of quick photodegradation, the photocatalytic process continues at a slower rate, indicating that the photocatalytic sites are further occupied by other CR molecules from solution. Thus, as the first adsorbed $\mathrm{CR}$ molecules are decomposed on the photocatalyst surface, other CR molecules are adsorbed on the active sites, with their almost complete removal occurring in only $50 \mathrm{~min}$ of the photocatalytic process. The results obtained were compared with data reported in literature which are included in Table 2. As observed from the data recorded in this table, the results obtained on $\mathrm{Cu}-\mathrm{bGu}-\mathrm{DS}$ complex compete with those obtained by Arora et al. by using $\mathrm{Cu}$-loaded $\mathrm{Fe}_{3} \mathrm{O}_{4} @ \mathrm{TiO}_{2}$ particles, when over $95 \%$ of $\mathrm{CR}$ in a $10 \mathrm{mg} / \mathrm{L}$ aqueous solution was photodegraded in $10 \mathrm{~min}$ of solar irradiation in an open space [40]. 

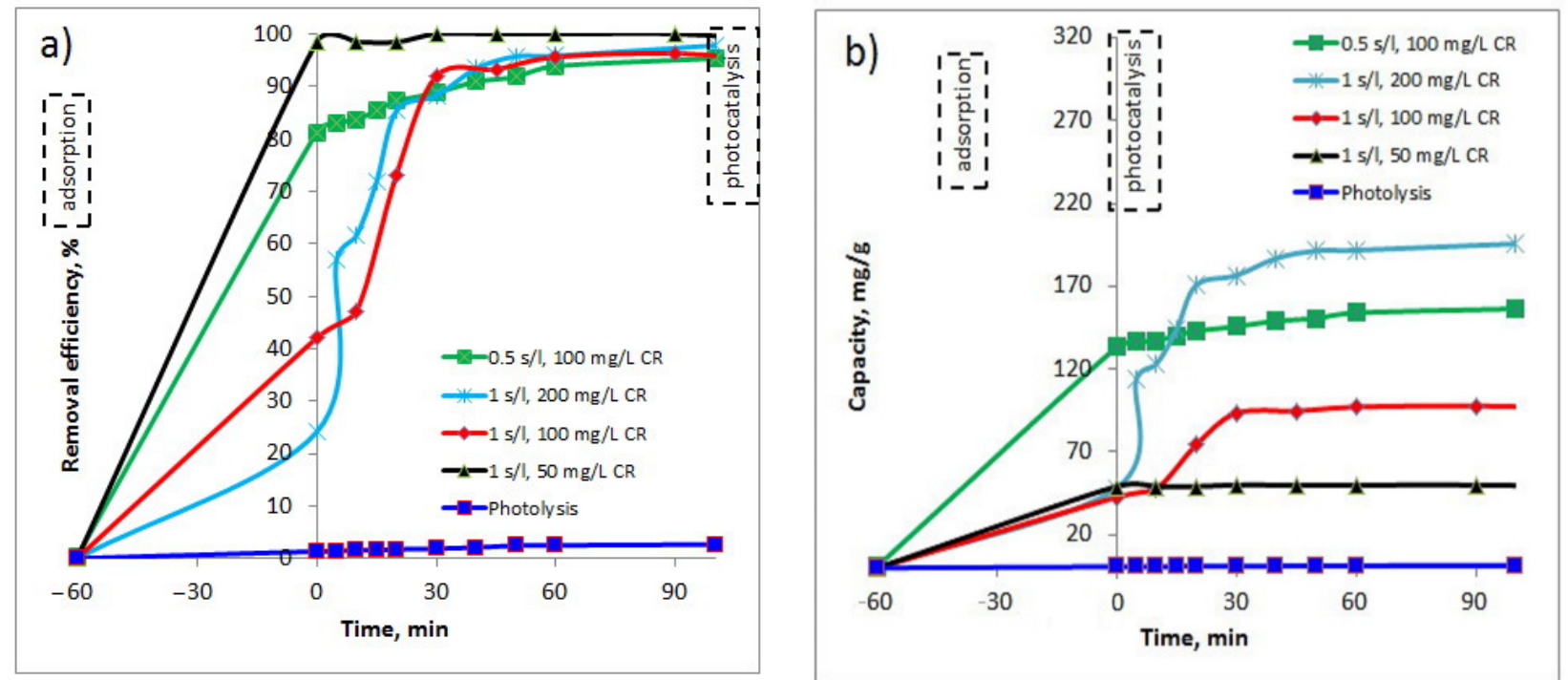

Figure 10. Removal efficiency (a) and photocatalytic capacity (b) of the synthesized Cu-bGu-DS complex in photodegradation of Congo Red (CR) dye.

Table 2. Photocatalytic performance of the prepared Cu-bGu-DS complex in comparison with literature.

\begin{tabular}{|c|c|c|c|c|c|}
\hline Photocatalyst & Time & Organic Pollutant & Light Wavelength & Efficiency (\%) & Ref. \\
\hline $\mathrm{PDMS} / \mathrm{TiO}_{2}$, coating & 6 days & $\begin{array}{l}\text { Methylene blue } \\
\text { (3.19 mg/L) }\end{array}$ & $365 \mathrm{~nm}$, natural & $100 \%$ & [18] \\
\hline PDMS-ZnO, powder & $50 \mathrm{~h}$ & $\begin{array}{l}\text { Phenol } \\
(1 \mathrm{mg} / \mathrm{L})\end{array}$ & $410 \mathrm{~nm}, 18 \mathrm{~W}$ & above $95 \%$ & [20] \\
\hline $\begin{array}{c}\mathrm{Mn}, \mathrm{Mo}, \mathrm{La} / \mathrm{TiO}_{2} / \mathrm{AC} \\
\text { powder }\end{array}$ & $5 \mathrm{~h}$ & $\begin{array}{l}\text { Reactive Red } 198 \\
\qquad(20 \mathrm{mg} / \mathrm{L})\end{array}$ & $\begin{array}{l}\text { Visible light Xenon } \\
\text { lamp, } 50 \mathrm{~W}\end{array}$ & $84 \%$ & [21] \\
\hline $\begin{array}{c}\mathrm{Cu} \text {-loaded } \mathrm{Fe}_{3} \mathrm{O}_{4} @ \mathrm{TiO}_{2} \\
\text { particles }\end{array}$ & $10 \mathrm{~min}$ & $\begin{array}{l}\text { Congo red } \\
(10 \mathrm{mg} / \mathrm{L})\end{array}$ & $\begin{array}{l}\text { Natural solar } \\
\text { irradiation }\end{array}$ & $95 \%$ & [40] \\
\hline Cu-bGu-DS complex & $50 \mathrm{~min}$ & $\begin{array}{l}\text { Congo red } \\
(200 \mathrm{mg} / \mathrm{L})\end{array}$ & $589.3 \mathrm{~nm}, 400 \mathrm{~W}$ & $>97 \%$ & This work \\
\hline
\end{tabular}

\section{Conclusions}

In this work, a new disiloxane ligand functionalized with 1,1,3,3-tetramethyl guanidine has been obtained and used to synthesize a copper(II) complex. The structure of both the ligand and its metal complex were established considering various spectral analyses (FTIR, NMR, XRF). The results obtained showed a good correlation with the theoretical calculations. Moreover, DSC investigation proved that the guanidine-functionalized disiloxane ligand and its $\mathrm{Cu}$ (II) complex are flexible supramolecular structures as they are characterized by negative glass transition temperatures, while their thermal stability was up to about $180^{\circ} \mathrm{C}$, as established by TG/DTG. The Cu-complex is characterized by light absorption in both UV and 500-900 $\mathrm{nm}$ spectral regions, and by a low value of the optical direct band gap energy $(2.9 \mathrm{eV})$, demonstrating a high activity in visible light-driven catalytic photodegradation of Congo Red dye (more than $97 \%$ in $50 \mathrm{~min}$ ). The results obtained are of great benefit for reducing the negative impact of economic activity on the environment, with applications in waste water remediation along the whole industrial chain starting from CR production and continuing with industrial sectors in which it is used, e.g., the textile industry. Further works are intended to verify the performance of siloxane-metal complexes in the photodegradation of other organic pollutants. 
Supplementary Materials: The following supporting information can be downloaded at: https:// www.mdpi.com/article/10.3390/polym14040817/s1, Section S1: Synthesis of bis(1,3-propyloxymeth yloxirane)disiloxane (DS-PMO). Section S2: Synthesis of bis-guanidine functionalized disiloxane (bGu-DS). Section S3: UV-Vis characterisation in DMF solution. Figure S1: Schematic representation of the synthesis of DS-PMO. Figure S2: Synthesis of bGu-DS. Figure S3: UV-Vis spectra of bGu-DS ligand and $\mathrm{Cu}-\mathrm{bGu}-\mathrm{DS}$ complex in $10^{-3} \mathrm{M}$ DMF solutions.

Author Contributions: Conceptualization, B.C.S. and V.H.; methodology, L.P.; software, D.P.; validation, V.H., M.I. and M.Z.; formal analysis, M.I., D.P. and M.Z.; investigation, M.E.F.; resources, V.H.; data curation, M.E.F.; writing—original draft preparation, M.E.F., L.P. and M.I.; writing—review and editing, visualization, supervision, B.C.S., M.I. and V.H. All authors have read and agreed to the published version of the manuscript.

Funding: This research received no external funding.

Institutional Review Board Statement: Not applicable.

Informed Consent Statement: Not applicable.

Acknowledgments: This work was supported from the budget of the Romanian Academy.

Conflicts of Interest: The authors declare no conflict of interest.

\section{References}

1. Rummi, D.S. Textile organic dyes: Polluting effects and elimination methods from textile waste water. Int. J. Chem. Eng. Res. 2017, 9, 121-136.

2. Al-Sakkaf, B.M.; Nasreen, S.; Ejaz, N. Degradation pattern of textile effluent by using bio and sono chemical reactor. J. Chem. 2020, 2020, 8965627. [CrossRef]

3. Katheresan, V.; Kansedo, J.; Lau, S.Y. Efficiency of various recent wastewater dye removal methods: A review. J. Environ. Chem. Eng. 2018, 6, 4676-4697. [CrossRef]

4. Stoilova, O.; Manolova, N.; Rashkov, I. Electrospun Poly(methyl methacrylate)/ $\mathrm{TiO}_{2}$ Composites for Photocatalytic Water Treatment. Polymers 2021, 13, 3923. [CrossRef] [PubMed]

5. Ainali, N.M.; Kalaronis, D.; Evgenidou, E.; Bikiaris, D.N.; Lambropoulou, D.A. Insights into Biodegradable Polymer-Supported Titanium Dioxide Photocatalysts for Environmental Remediation. Macromol 2021, 1, 201-233. [CrossRef]

6. Wang, Y.; Wang, Q.; Zhan, X.; Wang, F.; Safdar, M.; He, J. Visible light driven type II heterostructures and their enhanced photocatalysis properties: A review. Nanoscale 2013, 5, 8326-8339. [CrossRef]

7. Paria, S.; Reiser, O. Copper in photocatalysis. Chem CatChem 2014, 6, 2477-2483. [CrossRef]

8. Nicholls, T.P.; Bissember, A.C. Developments in visible-light-mediated copper photocatalysis. Tetrahedron Lett. $2019,60,150883$. [CrossRef]

9. Samira, S.F.; Carvalho, A.C.; Rodrigues, J.; Nakédia, F.L.; Carvalho, M.F. Photocatalytic degradation of dyes by mononuclear copper(II) complexes from bis-(2-pyridylmethyl)amine N,N,N-derivative ligands. Inorg. Chim. Acta 2020, $512,119881$.

10. Stanek, J.; Rösener, T.; Metz, A.; Mannsperger, J.; Hoffmann, A.; Herres-Pawlis, S. Guanidine metal complexes for bioinorganic chemistry and polymerisation catalysis. In Guanidines as Reagents and Catalysts II. Topics in Heterocyclic Chemistry; Selig, P., Ed.; Springer: Cham, Switzerland, 2015; Volume 51, pp. 95-164. [CrossRef]

11. Bienemann, O.; Hoffmann, A.; Herres-Pawlis, S. (Guanidine) copper complexes: Structural variety and application in bioinorganic chemistry and catalysis. Rev. Inorg. Chem. 2011, 31, 83-108. [CrossRef]

12. Boutevin, B.; Guida-Pietrasanta, F.; Ratsimihety, A. Side group modified polysiloxanes. In Silicon-Containing Polymers, The Science and Technology of their Synthesis and Applications; Jones, R.G., Ando, W., Chojnowski, J., Eds.; Kluwer Academic Publishers: Dordrecht, The Netherlands, 2000; pp. 79-112.

13. Missaghi, M.N.; Kung, M.C.; Kung, H.H. Polysiloxanes in catalysis and catalyst preparation: Opportunities for control of catalytic processes. Top. Catal. 2012, 55, 99-107. [CrossRef]

14. Pospiech, P.; Chojnowski, J.; Mizerska, U.; Makowski, T.; Strzelec, K.; Sienkiewicz, N. Polysiloxane microspheres functionalized with imidazole groups as a palladium catalyst support. Appl. Organometal. Chem. 2016, 30, 399-407. [CrossRef]

15. Cypryk, M.; Pospiech, P. Polysiloxanes as supports for catalysts. Chemik 2013, 67, 1173-1180.

16. Sosnin, I.M.; Vlassov, S.; Dorogin, L.M. Application of polydimethylsiloxane in photocatalyst composite materials: A review. React. Funct. Polym. 2021, 158, 104781. [CrossRef]

17. Cypryk, M.; Pospiech, P. Polysiloxanes as supports for transition metal catalysts. Polimery 2016, 61, 407-412. [CrossRef]

18. Wang, Y.; Huang, Z.; Gurney, R.S.; Liu, D. Superhydrophobic and photocatalytic $\mathrm{PDMS}_{\mathrm{TiO}}$ coatings with environmental stability and multifunctionality. Colloids Surf. 2019, A 561, 101-108. [CrossRef] 
19. Gerasimov, O.V.; Parmon, V.N. Photocatalysis by transition metal complexes. Russ. Chem. Rev. 1992, 61, 154-167. [CrossRef]

20. Sosnin, I.M.; Vlassov, S.; Akimov, E.G.; Dorogin, L. Transparent ZnO-coated polydimethylsiloxane-based material for photocatalytic purification applications. J. Coat. Technol. Res. 2020, 17, 573-579. [CrossRef]

21. Jorfi, S.; Mirali, S.; Mostoufi, A.; Ahmadi, M. Visible light photocatalytic degradation of azo dye and a real textile wastewater using $\mathrm{Mn}, \mathrm{Mo}, \mathrm{La} / \mathrm{TiO}_{2} / \mathrm{AC}$ nanocomposite. Chem. Biochem. Eng. Q. 2018, 32, 215-227. [CrossRef]

22. Huang, L.; Peng, F.; Yu, H.; Wang, H. Preparation of cuprous oxides with different sizes and their behaviors of adsorption, visible-light driven photocatalysis and photocorrosion. Solid State Sci. 2009, 11, 129-138. [CrossRef]

23. Lopez, J.B.; Holguin, N.F.; Gonzalez, J.C.; Sanchez, J.A.; Mitnik, D.G. Theoretical study of copper complexes: Molecular structure, properties and its application to solar cells. Int. J. Photoenergy 2013, 2013, 613064.

24. Nasrollahzadeh, M.; Akbari, R.; Sakhaei, S.; Nezafat, Z.; Banazadeh, S.; Orooji, Y.; Hegde, G. Polymer supported copper complexes/nanoparticles for treatment of environmental contaminants. J. Mol. Liq. 2021, 330, 115668. [CrossRef]

25. Litefti, K.; Freire, M.S.; Stitou, M.; González-Álvarez, J. Adsorption of an anionic dye (Congo red) from aqueous solutions by pine bark. Sci. Rep. 2019, 9, 16530. [CrossRef]

26. Pricop, L.; Fortună, M.E.; Popovici, D.; Asandulesa, M.; Racles, C.; Zaltariov, M.F.; Marangoci, N.; Savin, M.; Harabagiu, V. Nickel complexes of guanidine functionalized trisiloxane. J. Ing. Organomet. Polym. Mat. 2019, 29, 2024-2034. [CrossRef]

27. Liu, H.; Kobayashi, T.; Yu, H. Easy fabrication and morphology control of supramolecular liquid-crystalline polymer microparticles. Macromol. Rapid Commun. 2011, 32, 378-383. [CrossRef]

28. Zelenak, V.; Vargova, Z.; Gyoryova, K. Correlation of infrared spectra of zinc(II) carboxylates with their structures. Spectrochim. Acta Part A 2007, 66, 262-272. [CrossRef] [PubMed]

29. Racles, C.; Zaltariov, M.F.; Iacob, M.; Silion, M.; Avadanei, M.; Bargan, A. Siloxane-based metal-organic frameworks with remarkable catalyticactivity in mild environmental photodegradation of azo dyes. Appl. Catal. B Environ. 2017, 205, 78-92. [CrossRef]

30. Socrates, G. Infrared and Raman Characteristic Group Frequencies: Tables and Charts, 3rd ed.; A John Wiley \& Sons, Inc. Publication: New York, NY, USA, 2004; pp. 82-86.

31. Stewart, J.J. Computational Chemistry; Stewart Computational Chemistry: Colorado Springs, CO, USA, 2016. Available online: http:/ /OpenMOPAC.net (accessed on 2 July 2021).

32. Lee, C.; Yang, W.; Parr, R.G. Development of the Colle-Salvetti correlation-energy formula into a functional of the electron density. Phys. Rev. B Condens. Mater. 1988, 37, 785-789. [CrossRef]

33. Schmidt, M.W.; Baldridge, K.K.; Boatz, J.A.; Elbert, S.T.; Gordon, M.S.; Jensen, J.H.; Koseki, S.; Matsunaga, N.; Nguyen, K.A.; Su, S.; et al. General atomic and molecular electronic structure system. J. Comput. Chem. 1993, 14, 1347-1363. [CrossRef]

34. Schmider, H.L.; Becke, A.D. Optimized density functionals from the extended G2 test set. J. Chem. Phys. 1998, 108, 9624-9631. [CrossRef]

35. Merrick, J.P.; Moran, D.; Radom, L. An evaluation of harmonic vibrational frequency scale factors. J. Phys. Chem. A 2007, 111, 11683. [CrossRef] [PubMed]

36. Vlad, A.; Cazacu, M.; Zaltariov, M.F.; Shova, S.; Turta, C.; Airinei, A. Metallopolymeric structures containing highly flexible siloxane sequence. Polymer 2013, 54, 43-53. [CrossRef]

37. Zaltariov, M.F.; Cazacu, M.; Sacarescu, L.; Vlad, A.; Novitchi, G.; Train, C.; Shova, S.; Arion, V.B. Oxime-bridged mn6 clusters inserted in one-dimensional coordination polymer. Macromolecules 2016, 49, 6163-6172. [CrossRef]

38. Escobedo, M.A.; Ruiz-Lopez, I.I.; Ruiz-Peralta, M.d.L.; Tepech-Carrillo, L.; Sanchez-Cantu, M.; Moreno-Orea, J.E. Automated method for the determination of the band gap energy of pure and mixed powder samples using diffuse reflectance spectroscopy. Heliyon 2019, 5, e01505. [CrossRef] [PubMed]

39. Zanatta, A.R. Revisiting the optical bandgap of semiconductors and the proposal of a unified methodology to its determination. Sci. Rep. 2019, 9, 11225. [CrossRef] [PubMed]

40. Arora, P.; Fermah, A.; Rajput, J.K.; Singh, H.; Badhan, J. Efficient solar light-driven degradation of Congo red with novel $\mathrm{Cu}$-loaded $\mathrm{Fe}_{3} \mathrm{O}_{4} @ \mathrm{TiO}_{2}$ nanoparticles. Environ. Sci. Pollut. Res. 2017, 24, 19546-19560. [CrossRef] 